\title{
Gastric Adenocarcinoma with Superadded Candida Infection In A Chronic Alcoholic
}

\author{
Dr. Bhushan M. Warpe ${ }^{1}$,M.B.B.S., M.D. \\ Dr. Shweta S. Joshi' ${ }^{2}$,M.B.B.S., M.D. \\ ${ }^{1,2}$ Assistant Professor, Department of Pathology, B.K.L. Walawalkar Rural Medical College, \\ Ratnagiri, Maharashtra state, India.
}

\begin{abstract}
Esophagus is mainly reported to be involved by candidiasis in the gastro-intestinal tract with very rare occurrence in the stomach. Both benign and malignant gastric ulcers have been known to be associated with candidiasis. However the latter association is very rarely reported in literature. We present such a 'rare case' of an elderly chronic alcoholic/smoker who had epigastric tenderness without history of diabetes/hypertension. His GI endoscopy revealed a suspicious malignant gastric ulcer involving the greater curvature of the stomach. The 'gold standard' diagnostic tool of guided biopsy confirmed the diagnosis of gastric adenocarcinoma with superadded Candida infection. Anti-fungal medication followed by resection of the resectable tumor was the line of management.
\end{abstract}

Keywords: Alcoholic, gastric adenocarcinoma, gastric candidiasis

\section{Introduction}

In gastro-intestinal tract candidiasis, after esophagus, stomach and intestines are the rare sites involved. Gastric candidiasis is seen with altered gastric mucosal integrity like benign and malignant gastric ulcers, intake of corrosive chemicals, post-surgery, gastric perforation. Its association with benign gastric ulcers is more commonly seen compared to malignant ulcers. ${ }^{[1-3]}$ Although apart from diabetics, immunosuppressive patients like HIV positive cases, steroid usage, anti-cancer drugs, systemic diseases, organ and marrow transplantation, chronic alcoholism and smoking are the risk factors, gastric candidiasis have been reported in immunocompetent healthy cases too. ${ }^{[4]}$ Our rare case enlightens the definite diagnosis of gastric adenocarcinoma with superadded candida infection in a non-diabetic, chronic alcoholic with altered immune status.

\section{Case Report}

A 60-year-old gentleman came with complaints of pain in epigastrium and left hypochodrium since eight days. The pain was insidious in onset and was dull-aching type of pain. Patient also had increased frequency of urination at night. He had no history of nausea/vomiting/chest pain/fever/breathlessness/burning micturition/hematemesis/malena. Patient had no family history of similar complaints. He was not diabetic or hypertensive or on any immunosuppressive therapies. His personal history revealed he was a chronic alcoholic and chronic smoker since past 30 years.

On general examination he was afebrile, had a heart rate of 83 beats per minute and a blood pressure of 128/74 $\mathrm{mm} \mathrm{Hg}$. He had a respiratory rate of 17 cycles per minute.

Systemic examination revealed epigastric tenderness/discomfort, no guarding/rigidity and dark brown haeme negative stools. Rest of the physical examination was unremarkable. His lab investigations including complete blood counts, liver/thyroid/renal function tests, coagulation profile and urine analysis were normal.

Chest X-ray was normal. USG abdomen revealed a mass in left hypochondrium, probably as colonic mass without para-aortic lymphadenopathy, probably Gastro-intestinal stromal tumor (GIST).

USG prostate was suggestive of benign nodular hyperplasia of prostate. His serum PSA levels were within normal range $(<4 \mathrm{ng} / \mathrm{ml})$. USG-KUB was insignificant and showed normal findings.

Upper GI endoscopy (UGDscopy) showed a gastric fundus growth which was extending along the greater curvature of stomach. It was large ulcerative growth measuring $6 \mathrm{~cm}$ in diameter with thick necrotic, white exudates. UGDscopy-guided gastric mucosal growth biopsy was done.

We received multiple fragmented soft, white tissue bits, aggregating to size $0.2 \mathrm{~cm}$. On histopathology (Figure 1), sections studied revealed fragmented tiny biopsy showing a tumor infiltrating the fibrovascular tissue. The tumor was comprised of nests and clusters of cells surrounded by thin fibrovascular septae. The tumor cells were large, polygonal to round with scant vacuolated eosinophilic cytoplasm. The nuclei were ovoid, hyperchromatic with inconspicuous nucleoli. Some of the tumor cells had signet-ring cell appearance. The necrotic stroma showed chronic mononuclear infiltrate and showed fungal infiltration comprised of yeast 
and hyphal filaments (candida) invading the gastric tumor. Special stains of PAS (Figure 2) and Grocott methenamine silver/GMS (Figure 3) confirmed the fungal infection. On biopsy, diagnosis was given as adenocarcinoma with superadded candida infection.
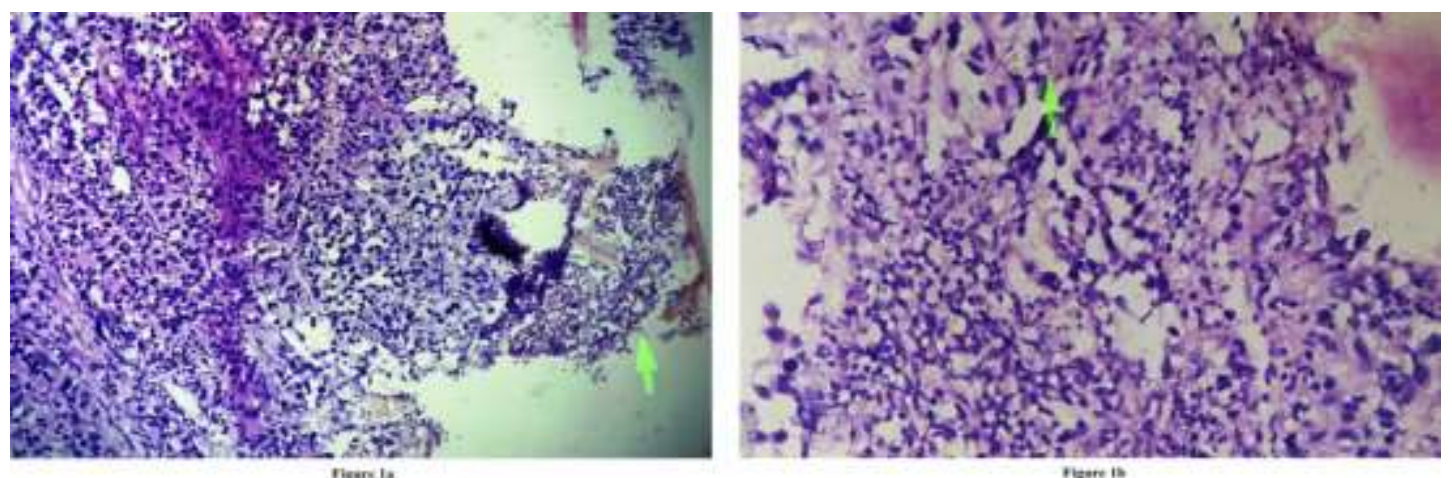

Figure 1a: Low power reveals gastric adenocarcinoma on biopsy comprised of nests and clusters of cells surrounded by thin fibrovascular septae. The necrotic inflammatory stroma showed fungal infiltration comprised of candidal yeast and hyphal filaments (arrow) invading the gastric tumor ( $\mathrm{H}$ and $\mathrm{E}, \times 100)$. Figure $1 \mathrm{~b}$ : High power revealed tumor cells which were large, polygonal to round with scant vacuolated eosinophilic cytoplasm. The nuclei were ovoid, hyperchromatic with inconspicuous nucleoli. Some of the tumor cells had signet-ring cell appearance (arrow) $(\mathrm{H}$ and $\mathrm{E}, \times 400)$.

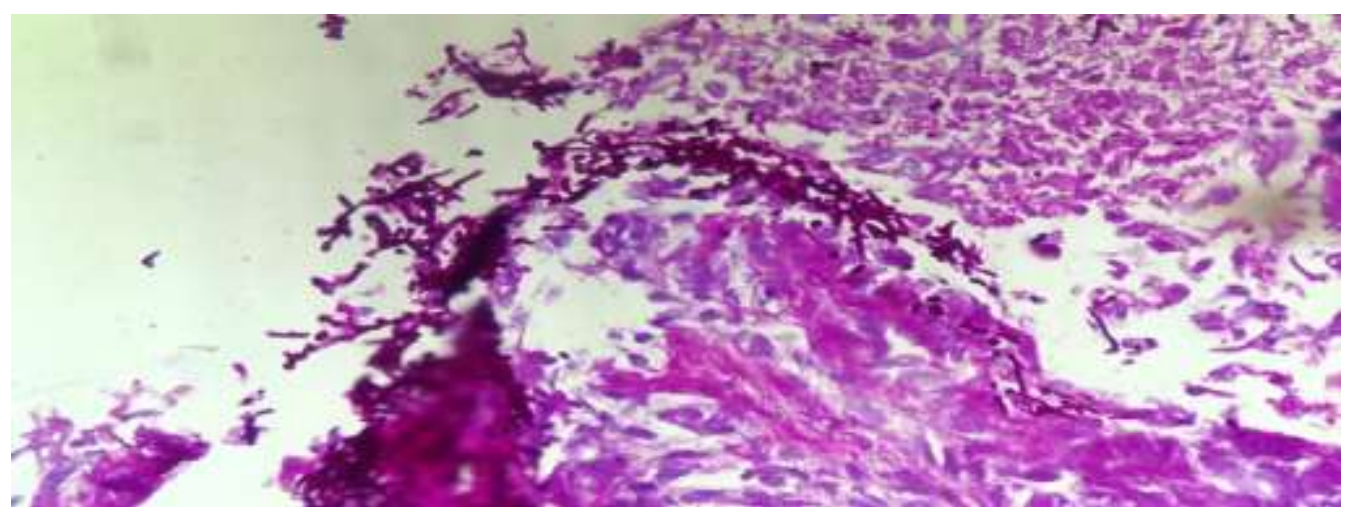

Figure 2: PAS staining of the candidal hyphal filaments and yeasts (PAS, $\times 400)$.

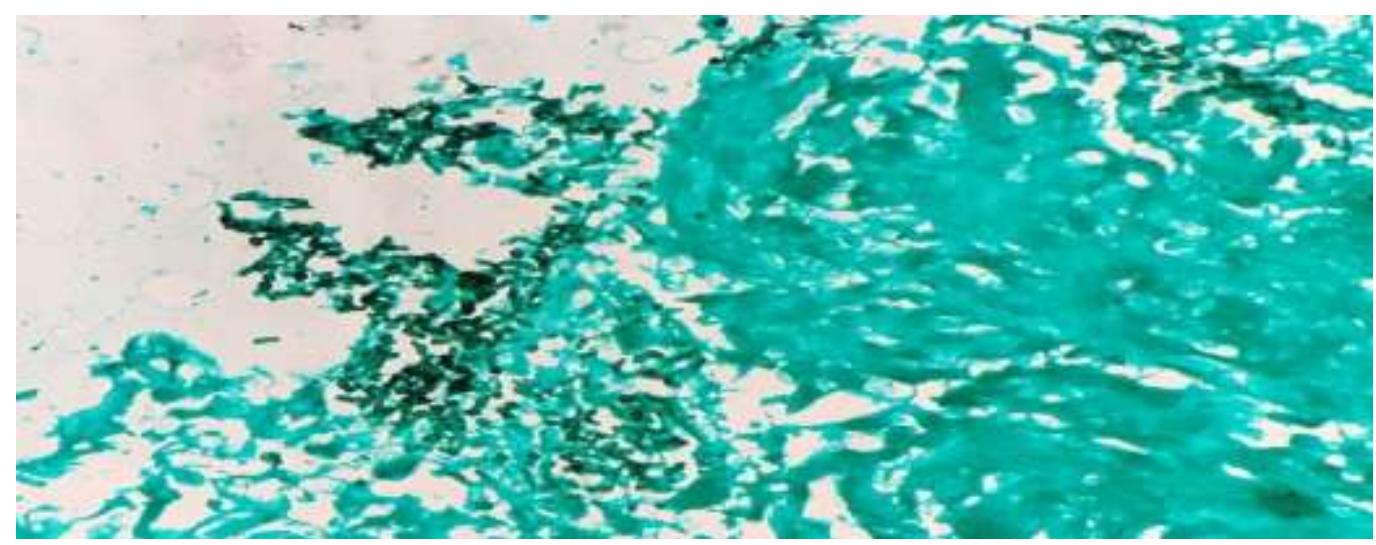

Figure 3: GMS staining of the candidal hyphal filaments and yeasts (GMS, $\times 400)$.

Additional work-up including hepatitis profile, T-lymphocytes counts, HIV test, VDRL-syphilis, antinuclear antibodies were normal while assessment of T-lymphocytes function by Mitogen stimulation test was abnormal suggested altered immune status.

The patient was given intra-venous anti-fungal medication before surgery of partial gastrectomy. Later patient was also given chemotherapy. The surgery was eventful and patient recovered well. She was later discharged on $10^{\mathrm{th}}$ post-operative day and on regular follow-up, she did not reveal any such similar prior complaints. 


\section{Discussion}

Association of gastric carcinoma with gastric candidiasis is very rare with few reported cases in world literature. ${ }^{[1]}$ Gastro-intestinal candidiasis is diagnosed by the demonstration of infiltration of tissue or ulcer slough by candidal yeasts and hyphal filaments. ${ }^{[4]}$ Candidal infection on the surface of gastric mucosa without infiltration must be ignored. ${ }^{[1]}$ As candida is a common commensal organism, its mere presence in culture studies or guided cytological smears must not suggest its gastric pathogenicity. ${ }^{[3]}$ The patients usually present with epigastric pain/tenderness, fever with chills, nausea/vomiting, abdominal mass. ${ }^{[2,3]}$ These malignant cases with superadded candida have a mean age of 59.7 years and tend to increase with increasing age. ${ }^{[3]}$

On gastric endoscopy, two types of lesions can be seen: thrush type with slough/exudates or ulcerative type with defined margins and a mean size of $66 \mathrm{~mm} \cdot{ }^{[3]}$ It helps in presuming a benign/malignant type of the gastric ulcer. However it is histopathology/biopsy, that is the gold standard. ${ }^{[1]}$ Biopsies usually show intestinal type of gastric adenocarcinoma with candidal infiltration of the tumor or ulcer slough. ${ }^{[1]}$ However our biopsy did not reveal neoplastic glands but rather neoplastic nests/clusters with polygonal tumor cells and also tumor cells with signet ring appearance. PAS and GMS special staining helps confirm the candidal superinfection. ${ }^{[1]}$ The latter was confirmed in our case.

Gingival candidiasis has been reported to occur in a Korean study in a case of gastric malignancy on chemotherapy, unlike our case. ${ }^{[6]}$ Recent studies quote the change in epidemiological pattern of the candida species:from C.albicans to non-albicans Candida species. ${ }^{[2]}$ Some known diabetics with psoriasis on chemotherapy can develop gastric ulcers by candida that can mimic malignancies on endoscopy, thus implying the importance of gastric biopsy. ${ }^{[4]}$ In an elderly individual, it is recommended that finding only candida on biopsy without malignancy must be considered as suspicious for the malignancy and must be rather better rebiopsied. ${ }^{[3]}$ Recurrent candida associated gastric ulcers at different times on follow-up is reported to occur very rarely. ${ }^{[5]}$ Thus inferring the importance of regular follow-up after any therapeutic intervention.

A clinical response implies relief of symptoms and reduction in the ulcer size on benign gastric ulcers. ${ }^{[3]}$ Also it is important to treat these benign gastric ulcer cases with anti-fungal medication because candida-associated benign ulcers are reported to have lower healing rates. However once healing is done by antifungal medication, candida are no longer detected. ${ }^{[3,5]}$ However the same does not hold true for malignant gastric lesions. The latter must be treated with definite surgical resection, if the tumor is resectable. ${ }^{[3]}$ Death of the gastric malignancy cases is not usually associated with systemic candidiasis. ${ }^{[3]}$ So before gastric surgery, few provide anti-fungal medication and few don't. ${ }^{[3]}$ However we recommend the usage of intra-venous fungal medication before the elective gastric resection.

\section{Conclusion}

UGDscopy helps in visualization of specific sites of gastric mucosal lesions. Histopathology is the 'gold standard' for the diagnosis of endoscopically detected gastric lesions and endoscopy is not complete without guided biopsy. Biopsy helps in early therapeutic decision and management of patients. Gastric adenocarcinoma with superadded candida infection is rare. It can be detected by H\&E stained slides but needs confirmation with special staining like PAS or GMS stained slides. Antifungal treatment followed by tumor resection is the optimum line of management for definitive cure of the resectable malignant gastric tumors.

\section{Financial support and sponsorship}

Nil.

\section{Conflicts of interest}

There are no conflicts of interest.

\section{References}

[1]. Subramanian KS, Gulia SP, Lavanya M, Arun Kumar SP. Gastric candidiasis with gastric adenocarcinoma intestinal type: A rare association. CHRISHMED J Health Res [serial online] 2015 [cited 2016 Feb 4];2:279-81.

[2]. Gugic D, Cleary T, Vincek V. Candida glabrata infection in gastric carcinoma patient mimicking cutaneous histoplasmosis. Dermatol Online J. 2008 Feb 28;14(2):15.

[3]. Jung MK, Jeon SW, Cho CM, Tak WY, Kweon YO, Kim SK, et al. Treatment of gastric candidiasis in patients with gastric ulcer disease: are antifungal agents necessary? Gut Liver. 2009;3(1):31-4.

[4]. Mansueto P, Pisciotta G, Tomasello G, Cabibi D, Seidita A, D'Alcamo A, et al. Malignant tumor-like gastric lesion due to Candida albicans in a diabetic patient treated with cyclosporin: a casereport and review of the literature. Clin Exp Med. 2012;12(3):201-5.

[5]. Sasaki K. Candida-associated gastric ulcer relapsing in a different position with a different appearance. World $\mathrm{J}$ Gastroenterol. 2012;18(32):4450-3.

[6]. Lim S, Kil TJ, Kim HR, Han S, Rha SY. A case of gingival candidiasis with bone destruction on gastric cancer patient receiving cytotoxic chemotherapy. Case Rep Oncol Med. 2014;2014:145394. 